\title{
LAAUTONOMÍA FINANCIERA: ¿CAUSA O EFECTO DE LA ESPECIALIDAD REGIONAL? ${ }^{1}$
}

\author{
Nicolò Megliorini \\ Università degli Studi di Verona
}

\begin{abstract}
Sumario. 1. Entre «forma» y «substancia»: la especialidad regional como categoría jurídico-constitucional. 2. Método comparado, especialidad regional y autonomía financiera. 3. Una comparación entre regímenes de financiación italiano y español: los modelos ordinarios... 4. ... y los especiales: País Vasco y Trentino-Alto Adige. 5. Especialidad regional y régimen común: la bilateralidad como «modelo». 6. El modelo de la bilateralidad: ¿Hacia su posible aplicación al régimen común? 7. Límites a la aplicación del modelo bilateral. 8. Posibilidades de evolución jurídica de la bilateralidad: interpretación y contradicciones.
\end{abstract}

\section{ENTRE «FORMA» $Y$ «SUBSTANCIA»: LA ESPECIALIDAD REGIONAL COMO CATEGORÍA JURÍDICO-CONSTITUCIONAL.}

La especialidad regional es un concepto muy importante para comprender las dinámicas y relaciones internas y exteriores en muchos ordenamientos jurídicos actuales. De hecho, solo a través de un análisis atento y completo de dicho concepto es posible apreciar los reflejos jurídicos que de ella se desprenden en los Estados compuestos contemporáneos. En cuanto a su naturaleza, la especialidad puede ser definida como una «categoría jurídicoconstitucional», mediante la cual se suelen definir algunos tipos de entidades regionales diferenciadas, y que concretan una forma específica de organización político-territorial del Estado.

Entre los criterios de clasificación de la especialidad regional que los constituyentes han utilizado para alcanzar a este resultado, hay que destacar el criterio que se puede calificar «nominalístico», que consiste en una individuación expresa y definitiva de las autonomías que van a ser comprendidas en esta clase. Ese criterio se encuentra utilizado, por ejemplo, por el legislador constitucional italiano (art. 116 de la Constitución italiana) y sirvió en 1948 para introducir en el ordenamiento la categoría de las Regiones de Estatuto Especial. El segundo es lo que encontramos en la Constitución española de 1978 y que podemos definir «substancial», con el cual el constituyente no determinó de forma expresa cuáles son las autonomías que van a pertenecer a este grupo; sino que reconoce en toda una serie de disposiciones constitucionales (Preámbulo, arts. 3-4-141-149, Disposición

${ }^{1}$ Comunicación presentada a la Jornada BiscayLaw II - El Poder normativo foral en un contexto multinivel y transnacional: propuestas y soluciones, Universidad de Deusto, 7 de marzo de 2013, <www.biscaylaw.deusto.es>. 
Adicional Primera y Tercera) la existencia de algunos rasgos determinados, típicos de algunas de ellas, que expresan una diferenciación cultural-política que determina una reglamentación distinta en algunas materias de estas Comunidades con respecto a las demás.

No obstante, este reconocimiento tiene que conciliarse con las previsiones de otras disposiciones del texto constitucional: primero con el artículo 2, que a pesar del reconocimiento de la garantía a la autonomía de «nacionalidades y regiones», decreta la «indisoluble unidad de la Nación española». ${ }^{2}$ Para comprender mejor este modelo de reconocimiento de la especialidad es necesario ofrecer una definición de lo que es el supuesto de la especialidad misma, es decir el concepto de «hecho diferencial»; esto es el elemento que permite a algunas CCAA poseer «caracteres particulares respecto al resto de las CCAA [...] que han generado una personalidad política diferenciada $\iota^{3}$. En realidad, está claro que también el criterio nominalístico del artículo 116 de la Constitución italiana vuelve a referirse a rasgos substanciales (la presencia de minorías étnicas y lingüísticas, situaciones de insularidad, etc.) que sin embargo han sido previamente validados y reglamentados por el constituyente mismo. La diferencia entre los dos ordenamientos consiste en el hecho que en Italia el constituyente hizo una «evaluación de especialidad» preventiva fundada sobre los hechos diferenciales, los cuales fueron recogidos y conservados en un nivel «nominalístico». En España estos hechos diferenciales son, entre otros, la presencia de una lengua propia, de instituciones intermedias, de una policía autonómica propia, etc.

Además de lo que se ha visto, uno de los hechos diferenciales más importantes en algunas CCAA es sin duda la existencia de los «derechos forales», cuyo origen se puede remontar a la Edad Media, con la diferenciación que se produjo en la incorporación del derecho romano en algunos territorios de la península ibérica. Además del derecho civil, la existencia de una tradición jurídica particular en estas regiones ha representado un hecho diferencial en el ámbito del derecho público, por el reflejo que ella ha tenido -entre los otros- en el ámbito de la financiación. Su origen histórico, que también se remonta a la Edad Media, ha tenido su actualización al final de las Guerras Carlistas, con el establecimiento del Concierto económico como continuación de los fueros anteriores. Entre los ámbitos de su mayor manifestación hay que destacar la existencia de una autonomía impositiva particular, basada en hechos históricos influyentes sobre la financiación (que aún siendo particular, tiene que desarrollarse con respeto al marco general

${ }^{2}$ La armonización con el primer principio fue realizada a través de una serie de fuentes de-constitucionalizadas, que han permitido la creación de un debate doctrinal muy interesante: Cruz-Villalon, P., «La Estructura del Estado o la Curiosidad del Jurista Persa», La Curiosidad del Jurista Persa, y otros Estudios sobre la Constitución, ed. CEPC, Madrid, 1999.

3 Aja E., El Estado Autonómico, ed. Alianza Editorial, Madrid, 2007, cit. p. 172. 
fijado en el dictado constitucional, lo que ha determinado la consiguiente desaparición de algunos institutos típicos del derecho foral histórico) ${ }^{4}$. La consecuencia a nivel constitucional de este estatus, es la peculiar modalidad de individualización del modelo de financiación del País Vasco y de Navarra: se trata verdaderos ejemplos de «reglamentación asimétrica», reflejo jurídico del hecho diferencial foral, fundamentada sobre el método de la introducción substancial.

\section{MÉTODO COMPARADO, ESPECIALIDAD REGIONAL Y AUTONOMÍA FI- NANCIERA.}

A pesar de todo, en una materia como la de la financiación autonómica -que tiene relaciones muy estrechas con el tejido político, económico e histórico de las sociedades- podemos recurrir al método comparado para realizar un análisis de su funcionamiento concreto. De hecho la «indagación comparativa» partiendo desde un «problema jurídico substancial» nos permite señalar analogías y diferencias entre institutos y sistemas pertenecientes a ordenamientos diferentes, denotar peculiaridades propias de un determinado sistema jurídico procediendo a una clasificación con finalidades cognitivas autónomas, y al final valorar la posibilidad de exportar determinados institutos para buscar soluciones y respuestas a dudas y problemas jurídicos específicos. ${ }^{5}$ En nuestro, caso el problema concreto al cual los ordenamientos constitucionales van dando soluciones -a veces análogas y otras diferentes- es que Estados diferentes, ante la perspectiva de elementos étnicos, forales, etc. que caracterizan alguna parte de sus territorios, prevén la creación de modelos de autonomías especiales que van exceptuando los regímenes ordinarios.

Siguiendo en una perspectiva comparada, es muy interesante referirnos a las Regiones de Estatuto Especial italianas, individualizadas -como vimos, de modo nominalístico- por el artículo 116 de la Constitución italiana. Estas cinco entidades regionales tienen todas una gran importancia en una investigación comparada con el ordenamiento español. Sin embargo, si queremos mantener el procedimiento en el ámbito de las modalidades generales de

${ }^{4}$ Por ejemplo, el derecho a no contribuir al sostenimiento de las cargas del Estado, la dispensa de participar obligatoriamente en el servicio militar (relevante antes la supresión del servicio militar obligatorio), los gravámenes particulares a la producción, etc.: Muñoz Machado S., Informe sobre España, ed. Crítica, Barcelona, 2012.

5 Bognetti G., «L'oggetto e il metodo», P. Carrozza, A. Di Giovine, G.F. Ferrari (eds.), Diritto costituzionale comparato, a cargo de, ed. Laterza, Roma-Bari, 2009, cit. p. 11. Para algunas aplicaciones del método de la comparación al tema de la financiación autonómica: Nicolini M., «Le relazioni finanziarie nel Belgio federale», Federalismo fiscale in Europa: Esperienze straniere e spunti per il caso italiano, ed. Edizioni Scientifiche Italiane, Napoli, 2012 y «Principio di connessione e metodo comparato», Federalismo fiscale: una sfida comparata, ed. Cedam, 2011. 
individuación formal del modelo de financiación, la que parece más interesante en una perspectiva comparada con el País Vasco, es sin duda la Región de Trentino-Alto Adige. Las razones de esta particular elección con respecto al País Vasco son sobre todo dos, una concerniente al aspecto institucional y la otra a las fuentes de derecho. En primer lugar, estas dos entidades regionales tienen una estructura político-territorial multinivel que parece análoga, y que se refleja en su plano institucional. De hecho, el País Vasco se estructura en los tres Territorios Históricos de Álava, Guipúzcoa y Vizcaya, mientras que la correspondiente división infra-regional está representada en Trentino-Alto Adige por las dos Provincias Autónomas de Trento y Bolzano. De todas formas, tenemos que dar cuenta de la existencia de algunas relevantes diferencias entre estos dos modelos de organización, por lo menos en el ámbito de la actualización de la asimetría.

En efecto, la existencia de las dos Provincias Autónomas está prevista directamente por la Constitución italiana en su artículo 116. Además de indicar cuáles son las Regiones de Estatuto Especial, el artículo sigue afirmando que la Región de Trentino-Alto Adige/Südtirol está constituida por las dos Provincias Autónomas de Trento y Bolzano. Además la Constitución deja la implementación de las competencias de las autonomías regionales a los Estatutos, que sin embargo van a ser aprobados por el Parlamento con una ley constitucional. La consecuencia normativa de estos principios constitucionales es que, en el desarrollo del nivel autonómico provincial, las dos Provincias Autónomas han conseguido recoger las mismas competencias legislativas y administrativas de las Regiones (en los límites fijados por el Estatuto), aunque hay que precisar que las Provincias Autónomas tienen claramente, por su estructura- competencias diferentes de las que tienen las otras autonomías especiales. Además, entre sus competencias «ordinarias» hay capacidades de gastos en ámbitos que tal vez no pertenecen a las otras entidades especiales italianas, aunque caracterizadas por el mismo nomen juris. ${ }^{6}$ Este carácter muy peculiar de la estructura institucional autonómica del Trentino-Alto Adige ha sido definido por la doctrina como una verdadera forma de «especialidad en la especialidad» ${ }^{7}$. En el País Vasco, aunque el resultado final es análogo en lo que se refiere a la división entre tareas y competencias, la implementación ha seguido un camino diferente: las instituciones intermedias entre la CA y los municipios encuentran un reconocimiento constitucional al «amparo y el respeto de los derechos históricos de los ter-

${ }^{6}$ Por ejemplo, la competencia financiera de la Provincia Autónoma de Trento sobre sus Universidades (art. 3 decreto legislativo 18 luglio 2011, n. 142) y las competencias entre las políticas de ayudas sociales (art. 8 c. 1.25 Estatuto Trentino-Alto Adige): PArolari S., Valdesalici A., «La provincia autonoma di Trento. Verso il consolidamento dell'autonomia finanziaria», Le istituzioni del federalismo 1/2012, pp. 103-104.

7 Palermo F. «Federalismo fiscale e Regioni speciali: vecchi nodi vengono al pettine», Le istituzioni del federalismo 1/2012, p. 15. 
ritorios forales» (Disposición Adicional Primera). Pero es el Estatuto el que sigue afirmando y garantizando las autonomías, decretando, en su artículo 2, que «el territorio de la Comunidad Autónoma del País Vasco quedará integrado por los Territorios Históricos que coinciden con las provincias, en sus actuales límites, de Álava, Guipúzcoa y Vizcaya [...]». El Estatuto mismo, en modo parecido al caso del Trentino-Alto Adige, dispone cuáles son las competencias normativas (exclusivas, de desarrollo normativo y ejecutivas) de los territorios forales en su capítulo IV del Título II. Además, (y análogamente al Título VI del Estatuto del Trentino-Alto Adige) fija en el Título III una reglamentación muy precisa de las competencias financieras de los Territorios Históricos, de las aportaciones y de las relaciones entre la CA y las diputaciones forales, que se traduce en el hecho de que las diputaciones mismas «establecen, recaudan y gestionan directamente la mayoría de los impuestos de los ciudadanos vascos $»^{8}$. El marco se completa con la Ley de Territorios Históricos (Ley 27/1983), que determina la relaciones entre las instituciones comunes de la CA y los órganos forales y que representó una de las razones de la división de los partidos vascos PNV y Eusko Alkatarsuna en los años ochenta.

\section{UNA COMPARACIÓN ENTRE REGÍMENES DE FINANCIACIÓN ITALIA- NO Y ESPAÑOL: LOS MODELOS ORDINARIOS...}

El segundo perfil de gran transcendencia es la posibilidad de exceptuar el régimen ordinario de financiación, que en ambos casos la especialidad permite, sobre todo en el sistema de las fuentes. Antes que analizar las modalidades de la excepción a dicho modelo ordinario, hay que explicar el procedimiento de su individualización en ambos ordenamientos. En España, la disciplina está ante todo definida por los artículos de la Constitución, que van a constituir la así llamada Constitución financiera (artículos 133.1149.1.14-156-157-158), de régimen ordinario. El artículo 157 determina precisamente los recursos de las CCAA, pero deja al Parlamento la obligación de definir con una ley orgánica (la LOFCA) el marco y los limites de la autonomía.

La LOFCA en su primer artículo dispone que «la financiación de las Comunidades Autónomas se regirá por la presente Ley Orgánica y por el Estatuto de cada una de dichas Comunidades»: los Estatutos tienen que ser compatibles con las normas constitucionales y por eso, con la LOFCA. A pesar de todo, debemos señalar que en el régimen ordinario la individualización del modelo no es estrictamente unilateral, sino multilateral. En efecto, las cuestiones financieras comunes a las CCAA son negociadas en el Consejo de Política Fiscal y Financiera, cuyos acuerdos se trasladan normalmente al contenido de la LOFCA y sus modificaciones. Paralelamente, en Italia el régimen ordinario se basa sobre el dispuesto de la Constitución, que explica

${ }^{8}$ Aja E., El Estado Autonómico, ed. Alianza Editorial, Madrid, 2007. 
también sus principios de carácter general en el artículo 119 (recientemente modificado por la ley constitucional 1/2012). Además de los principios generales, la regulación de la materia de la financiación se determina por una ley estatal, que actualmente es la ley 42/2009, que aplica el así llamado principio del «federalismo fiscal»; esa ley ha delegado en el Gobierno su implementación, que ha sido llevado a cabo a través de algunos decretos legislativos, que definirán los principios y detalles de la nueva normativa financiera.

Entre los rasgos principales del nuevo federalismo fiscal se encuentra el distanciamiento con respecto al modelo anterior del sistema de la financiación derivada, con la previsión de dos instrumentos principales de obtención de recursos por las Regiones ordinarias: participaciones en tributos estatales (art. 7 c.1 letra a ley 42/2009); tributos propios (art. 7 c. 1 letra b), que suelen dividirse entre tributos propios derivados (introducidos por leyes estatales), y tributos propios instituidos por las Regiones con normas autónomas, respetando la prohibición de la doble imposición fiscal. ${ }^{9}$ Volviendo al análisis, el primer elemento que podemos advertir es, por supuesto, la analogía en el papel de la multilateralidad en la individualización del modelo ordinario de ambos los ordenamientos. El segundo se refiere a las fuentes mismas y se traduce en una diferencia entre los dos modelos: en España la individualización se implementa con una fuente reforzada, es decir la LOFCA; en Italia (por su sistema de fuentes) se trata de una ley ordinaria del Estado, que ha desarrollado directamente una norma constitucional que fija por si misma principios generales aunque de carácter detallado (art. 119).

\section{4. ... Y LOS ESPECIALES: PAÍS VASCO Y TRENTINO-ALTO ADIGE.}

En las autonomías especiales que se analizan, el proceso de organización del modelo de financiación es diferente y supone una excepción a las relaciones entre las fuentes del derecho con respecto a la normativa ordinaria. La diferenciación puede ser resumida con la palabra «bilateralidad». En el País Vasco esta bilateralidad se conecta con el instituto del Concierto Económico que «constituye la pieza esencial en la ordenación de las relaciones financieras y tributarias entre el Estado y esta Comunidad autónoma» y que «es la expresión del derecho histórico que a día de hoy posee mayor repercusión y relevancia». El marco de las fuentes está representado en el País Vasco ante todo, por supuesto, por la Constitución, aunque ni la disposiciones que atribuyen poder tributario a las CCAA (arts. 133.2-156-157), ni las que lo confieren a las Corporaciones Locales (arts. 133.2-140-142) son aplicables a estas instituciones vascas. El principio constitucional en este caso está fijado, como vimos, por la Disposición Adicional Primera, que «ampara y respeta los derechos históricos de los territorios forales», entre los cuales

${ }^{9}$ La Scala A.E., «L'attuazione del cd. «federalismo fiscale» nei rapporti Stato-Regioni», Federalismo fiscale e autonomia degli enti territoriali, ed. G. Giappichelli, 2010. 
encontramos también la regulación de la materia tributaria. Esta previsión se desarrolla por el Titulo III del Estatuto del País Vasco (sobre Hacienda), que en su artículo 41.1 afirma que «las relaciones de orden tributario entre el Estado y el País Vasco vendrán reguladas mediante el sistema foral tradicional de Concierto Económico o Convenios». El mismo artículo 41 sigue diciendo que el Concierto se aprueba por ley, de modo que la «forma de ley concede también al texto concertado vigente en cada momento una protección y jerarquía de la que no gozan las disposiciones reglamentarias» ${ }^{10}$. Un elemento muy importante es el papel de la LOFCA en todo este sistema de reglamentación: su vigencia permanece, pero sin embargo tenemos que considerarla de naturaleza subordinada o supletoria y será aplicada en todo lo que se emane con base en el instrumento del concierto. Por lo que concierne a la reglulación de la Hacienda y sus tributos debemos señalar numerosos métodos de obtención de recursos: ante todo, aportaciones de las Diputaciones Forales ${ }^{11}$, impuestos propios de la CA y finalmente, otros ingresos del artículo 42 del Estatuto ${ }^{12}$. Es muy interesante evidenciar cómo el Concierto Económico va directamente a reglamentar estas formas de recursos; así las cosas, el Estado no puede fijar cesiones y recargos sobre impuestos estatales. Además de estas previsiones, el País Vasco transfiere a través del «Cupo», parte de los recursos tributarios al Estado central, para hacer frente a las cargas generales de competencia exclusiva del Estado. El órgano bilateral donde se produce el acuerdo es la Comisión Mixta, constituida por doce miembros; el Concierto vigente está regulado por la Ley 28/2007.

En Trentino Alto-Adige también la negociación del modelo aporta modificaciones estructurales a la normativa fijada para las autonomías ordinarias. En este caso encontramos, además de la Constitución con el artículo 116, el Estatuto, que establece el modelo financiero de la Región. Este ha sido modificado muchas veces, la última de las cuales ha sido efectuada por el así llamado «Acuerdo de Milán». Este acuerdo, firmado por el Gobierno, las dos Provincias Autónomas y la Región el 30 de noviembre de 2009, fue introducido en la ley 191/2009, es decir la Ley Finaciera de 2010, modalidad que como veremos encuentra su base jurídica en el art. 104 del Estatuto de Autonomía. Otra particularidad es que a este marco (como a lo de las otras Regiones de Estatuto Especial) no se le aplica la regulación ordinaria delineada por la ley 42/2009, excepto por lo que concierne tres normas, es decir los artículos 15 (ciudades metropolitanas), 22 (reparto equitativo de

${ }^{10}$ Rodrigo Ruiz M. A., Las fuentes del derecho financiero en la CA del País Vasco, Editoriales del Derecho Reunidas, Madrid, 2006, cit. pp. 28,171,172.

${ }^{11}$ Las Diputaciones se obligan a transferir al Gobierno autonómico los importes necesarios para el sostenimiento de las cargas generales del País Vasco no asumidas por los Territorios Históricos.

12 Por ejemplo, transferencias de Fondo de Compensación Interterritorial y ingresos de derecho privado. 
infraestructuras) y sobre todo el artículo 27. Este artículo determina la participación de las dos Provincias Autónomas al alcance de los objetivos de equidad y solidaridad y al ejercicio de los derechos y obligaciones que de ellos derivan. El aspecto importante que percibimos analizando los ingresos financieros es la presencia de una autonomía financiera muy fuerte de las Provincias mismas; por ejemplo, en la Provincia Autónoma de Trento los ingresos están compuestos por la participación en tributos estatales, a través de dos mecanismos: la «cuota fija» (fijada por el Titulo VI del Estatuto regional, representa la mayoría de las entradas locales) y la «cuota mutable» (negociada cada año por las dos P. A. y el Estado). El último mecanismo es lo de las transferencias estatales, aunque ahora componen una mínima parte de los ingresos generales. ${ }^{13}$

\section{ESPECIALIDAD REGIONAL Y RÉGIMEN COMÚN: LA BILATERALIDAD COMO «MODELO».}

Un análisis comparado de los dos sistemas normativos de País Vasco y Trentino-Alto Adige permite descubrir numerosas analogías, aunque el sistema ordinario, como hemos visto, ha sido implementado por medidas diferentes. En primer lugar es fundamental el rol de la bilateralidad que exceptúa la reglamentación ordinaria y las fuentes que la introducen prescindiendo de las normativas ordinarias. En el caso del País Vasco es una norma del Estatuto (es decir una norma aprobada con Ley Orgánica estatal) la que desarrolla de este modo una típica competencia jurídica de los derechos forales, es decir la financiación. En Trentino-Alto Adige la particularidad, mucho más original, está prevista por el artículo 104 del Estatuto: las modificaciones a las normas sobre la financiación pueden ser aportadas con ley ordinaria del Estado a iniciativa conjunta del Gobierno y la Región (o de la Provincia si ella está ejercitando sus competencias). Es una forma real de negociación bilateral para modificar una norma estatutaria de rango constitucional, como pasó por ejemplo en 2009 con el Acuerdo de Milán. La originalidad de este modelo consiste en el hecho de que un acuerdo que representa una forma de protección de la capacidad de negociación de las autonomías «subroga la garantía representada de la forma legislativa constitucional ${ }^{14}$ del Estatuto $\mathrm{y}$ 《puede funcionar de parámetro de legitimidad constitucional» ${ }^{15}$. Lo que se ha hecho es una desconstitucionalización del modelo de financiación en esta Región que suele implementar la bilateralidad misma; una inversión de fuentes que en España no existe.

13 Parolari S., «La provincia autonoma di Trento. Verso il consolidamento dell'autonomia finanziaria», Le istituzioni del federalismo 1/2012, pp. 115-124.

14 Pedrazza Gorlero M., «L'ordine frattalico delle fonti del diritto», ed. CEDAM, Padova, 2012. Cit p.41.

15 Sentencia de la Corte Constitucional 323/2011 de día 21 noviembre, FJ 2.3 cons. der. 
Como se ha visto, la disciplina ordinaria en ambos ordenamientos conduce a formas de negociación, pero multilaterales. En Italia, hay dos formas de negociación multilateral: una en el principio de la actualización de la ley 42/2009, con la presencia de una Conferencia Unificada para determinar el contenido de los decretos legislativos; otra al final de la nueva normativa, que una vez ejecutada, sigue disponiendo en su artículo 18 un «Pacto de Convergencia» (negociado en la misma Conferencia) para la determinación del gasto «estándar» que cada Región prevé. En España, la negociación multilateral suele presentarse en el acuerdo para reformar la LOFCA en el Consejo de Política Fiscal y Financiera. Es decir, en Italia actualmente la multilateralidad es necesaria para la actualización de principios previstos por el legislador estatal, mientras que en España, más ampliamente, va a definir el mismo contenido substancial del modelo de financiación ordinario.

\section{EL MODELO DE LA BILATERALIDAD: ¿HACIA SU POSIBLE APLICA- CIÓN AL RÉGIMEN COMÚN?}

En conclusión, analizado el complejo de relaciones que la especialidad introduce, tenemos que reflexionar sobre los efectos mismos del cambio que la bilateralidad aporta en ambos Estados. En un primer análisis, la amplitud de su eficacia puede ser considerada equivalente en los dos ordenamientos. De hecho, hay una regulación ordinaria que prevé formas de negociación multilateral (que se reflejan en la LOFCA por un lado, en la ejecución de la ley de delegación por el otro) tanto en España como en Italia, que representan el modelo para las autonomías que no tienen el reconocimiento constitucional especial; la cual se encuentra exceptuada por un conjunto de fuentes que configuran una negociación bilateral en un contexto territorial multinivel. Esta negociación, implementada con leyes del Estado, se manifiesta conjuntamente con una normativa substancial que fija modelos de financiación particular (poder de establecimiento, recaudación y gestión de la mayoría de los impuestos por los Territorios Históricos del País Vasco, presencia de un sistema de relaciones entre la Región y las Provincias -que permiten a las Provincias mismas una fuerte autonomía en el aspecto de la financiación- en el Trentino-Alto Adige).

No obstante, tenemos que mencionar una tendencia que, paralelamente al debate sobre el federalismo fiscal en Italia, se ha desarrollado en España aproximativamente desde 2005. Esta tendencia comienza con la reforma del Estatuto catalán en 2006, donde sobre todo (entre las múltiples medidas sobre la financiación de Cataluña), se concretaron límites para la participación de Cataluña en los mecanismos de solidaridad dispuestos por la Constitución misma; además se previó un papel fundamental del principio de bilateralidad, que representaba la forma preferencial para la concreción del modelo de financiación. El principio se manifestaba en las actividades de la Comisión bilateral Estado-Generalitat y la Comisión de asuntos fiscales y económicos, con una siguiente marginalización del sistema multilateral con 
respecto a Cataluña. La sentencia 31/2010 anuló el alcance de estas alteraciones, reafirmando la relación Estatuto-LOFCA en la determinación del modelo de financiación ordinario.

No obstante, el debate siguió y la Generalitat de Cataluña empezó reclamar un sistema fiscal basado en los principios del concierto vasco. El 4 octubre de 2011 el Instituto de Estudios Autonómicos (IEA) publicó el «Informe sobre la aplicación a Cataluña de un nuevo modelo de financiación basado en el concierto económico», que expresaba la reclamación catalana de un Pacto Fiscal con el Estado español. El hecho diferencial que reclama Cataluña en el ámbito fiscal se basa sobre su economía, como la especialidad del tejido productivo y industrial catalán, el número de población inmigrante, los costos de mantenimiento de infraestructuras, etc., y además se reclaman, entre las otras, la exacción, gestión, recaudación y liquidación de todos los tributos soportados en la CA, por la Agencia Tributaria de Cataluña. Es decir, la Comunidad tiene atribuida la gestión de la casi totalidad de los tributos, y retorna al Estado una parte de los ingresos obtenidos, para contribuir a las cargas del Estado no asumidas por la Comunidad Autónoma; y, lo más importante por nuestro análisis, este retorno se fija bilateralmente. Esta regulación financiera para Cataluña no es la prevista y el sistema sigue con la negociación multilateral prevista por la LOFCA y el Estatuto (interpretado por el Tribunal Constitucional); pero si se aprobase, ¿cuáles serían los reflejos jurídicos sobre el ámbito de las fuentes que hemos analizado hasta ahora desde una óptica comparada? La contestación es muy simple: si el sistema de la individuación y reforma del modelo de financiación llegara a ser bilateral para todas la CCAA, la excepción al sistema ordinario de las fuentes será mucho más perceptible en Italia con respecto a España.

\section{LÍMITES A LA APLICACIÓN DEL MODELO BILATERAL.}

Para completar el análisis, hay que analizar la posibilidad de esta evolución jurídica, es decir si el modelo del concierto vasco puede ser extendido a otras CCAA o exportado a otros ordenamientos que tienen dinámicas de recursos diferentes; en definitiva, con respecto a nuestra comparación, analizar si esta extensión puede exceptuar el artículo 158 de la Constitución de 1978 y el artículo 119 de Constitución italiana (modificado por la ley constitucional 1/2012). Para contestar hay que considerar que, a pesar de todo, el papel de la bilateralidad (aún fundamental en España y en Italia) no puede desarrollarse sin limitación alguna: en caso italiano un ejemplo muy reciente de los límites a la bilateralidad en las Regiones de Estatuto Especial lo encontramos en la sentencia de la Corte Costituzionale italiana 102/2008; decisión muy importante porque el Tribunal Constitucional italiano, además de definir los límites de la potestad tributaria autonómica con respecto al dictado constitucional, ha introducido otra limitación para el legislador regional: la normativa europea la cual, aún no teniendo competencias en materia fiscal, puede integrarse también en este ámbito con los ordenamientos nacio- 
nales en cuanto que tiene como objetivo garantizar la libertad de prestación de servicios y la prohibición de ayudas de Estado.

Lo que se ha producido es una «erosión» de las competencias nacionales en esta misma tarea, en este caso referida a la potestad impositiva de la Región de Estatuto Especial de Cerdeña. En el ordenamiento español el alcance reformador tampoco está desprovisto de límites. De hecho, la bilateralidad no puede derogar ni la transferencia de una parte de los recursos de una CA a otra por el Estado según la norma del artículo 158 que dispone el equilibrio y la compensación interterritorial; ni tampoco el principio de solidaridad afirmado por los artículos 2 y 138.1 de la Constitución. Estos son principios constitucionales que, como sabemos, no están comprendidos entre los artículos que la Constitución misma permite ser excepcionados por la regulación establecida en el marco de la especialidad. Además es el Concierto mismo el que va a delimitar potestades financieras y normativas de la CA y los Territorios Históricos, de hecho hay un control del Estado en la negociación para el respeto de estos límites también.

A la postre, hay que dar cuenta de los límites de tipo jurisdiccional. Se puede mencionar la sentencia de la Sección Segunda de la Sala Tercera (de lo Contencioso Administrativo) del Tribunal Supremo de 9 de diciembre de 2004 relativa a las normas forales de los Territorios Históricos que regulaban sus respectivos Impuestos de Sociedades; la decisión es muy importante porque, una vez más, se pronuncia sobre los límites de las normas forales en un contexto multinivel, que se crea con la aplicación de la normativa europea. En esta sentencia el Tribunal declaró incompatible con la prohibición de «ayudas de Estado», la regulación de los Territorios Históricos sobre el Impuesto de Sociedades. Esta decisión plantea un doble conjunto de problemas: en primer lugar, un problema en relación a la legitimación activa para la impugnación de estas normas, «permitiéndose prácticamente a cualquiera que alegue un «interés legítimo» y sin muchos más requisitos, impugnar en los Tribunales contencioso-administrativos cualquier norma foral de esta índole. El asunto ha encontrado una solución con la reforma de LOTC de 2007, que ha reforzado la protección de las normas fiscales de los territorios forales, reservándoles una regulación parecida a las leyes ordinarias, impidiendo su impugnación ante los tribunales ordinarios. El segundo problema es más interesante para nuestro análisis y consiste en la decisión misma del Tribunal Supremo de anular la diferenciación producida por las normas de los Territorios Históricos en materia fiscal; «si los Territorios Históricos al regular los impuestos concertados de normativa propia no pueden apartarse de la regulación del Estado, ¿dónde queda su autonomía normativa, garantizada expresamente por el Concierto Económico? ${ }^{16}$. La cuestión fue aclarada por el Tribunal de Justicia de la Co-

16 LarrazÁbal BasáÑEz S., «Los retos del Concierto Económico Vasco tras la Sentencia del Tribunal de Justicia de las Comunidades Europeas de 11 de Septiembre de 2008», Boletín JADO. Bilbao. Año VII. No 17. Septiembre 2009, cit. pp. 40-41. 
munidad Europea que en el famoso «caso Azores» fijó los requisitos por los cuales un régimen fiscal diferenciado puede ser compatible con la prohibición de ayudas de Estado ${ }^{17}$. Estos principios fueron implementados por el mismo Tribunal de Justicia en la sentencia del 11 septiembre 2008, que decidió sobre la cuestión prejudicial relativa al régimen vasco.

No podemos finalizar sin mencionar otros modelos de asimetría que existen en la Constitución española: el navarro y el canario. El sistema navarro, como el del País Vasco, se basa sobre el hecho diferencial de la existencia de un derecho foral histórico que influyó la materia de la Hacienda también. En Navarra su concreción fue realizada por el Estatuto de Autonomía (LORAFNA), que desarrollando la Primera Disposición Constitucional, tradujo esta especialidad en un sistema análogo al País Vasco, con la presencia de un Convenio negociado con el Estado y de una Aportación que transfiere parte de los recursos que corresponden a las cargas del Estado mismo. Hay también que mencionar la diferente estructura territorial de Navarra, por ser uniprovincial, que difiere de la división provincial vasca en diferentes territorios y de su carácter multinivel.

Por lo que concierne las Canarias, que representan una forma de especialidad aun perteneciendo al sistema ordinario mismo, hay que partir de lo dispuesto en la Disposición Adicional Tercera de la Constitución Española. La especialidad se representa aquí claramente por una situación de insularidad, que produce una regulación basada en la presencia de una forma de financiación que podemos también definir concertada. El informe previo del Parlamento canario no es vinculante y, por tanto, las Cortes podrían reformar el régimen de Canarias aunque la opinión del Parlamento fuera contraria; no obstante las Cortes tienen la obligación de pedir este informe al Parlamento canario, como estableció una sentencia del Tribunal Constitucional de 1984 (que anuló un decreto-ley estatal que modificaba un elemento de fiscalidad canaria porque carecía del informe). También el régimen canario, por su naturaleza derogatoria y por la presencia de algunas normas substanciales que establecen beneficios tributarios, ha tenido que enfrentarse con el derecho europeo (un elemento ejemplar es el Estatuto especial para Canarias, recogido en el Acta de Adhesión de España a la Comunidad Europea, que prevé formas de protección por ser Canarias una región «ultra-periférica»).

17 Estos requisitos son: a) que la autoridad regional o local cuente con un estatuto político y administrativo distinto del Gobierno Central (autonomía política o institucional) ; b) que la decisión haya sido adoptada sin que el Gobierno Central haya intervenido directamente en su contenido (autonomía procesal); c) que las consecuencias financieras de un tipo impositivo reducido (es decir, la reducción de ingresos que ello supone para dichas autoridades), no se vean compensadas por ayudas o subvenciones procedentes de otras regiones o del Gobierno Central (autonomía económica). 


\section{POSIBILIDADES DE EVOLUCIÓN JURÍDICA DE LA BILATERALIDAD: INTERPRETACIÓN Y CONTRADICCIONES.}

Finalmente, una vez analizados todos los contextos necesarios para evaluar la extensión del modelo concertado a entidades regionales donde no hay elementos diferenciales en materia financiera, la contestación a nuestra pregunta tiene que ser, podemos decir, «de compromiso». Podría llevarse a efecto la extensión del modelo de financiación vasco a Cataluña, siempre que se lograse conciliar el principio de la especialidad bilateral con el respeto a la solidaridad y la coordinación con el Estado central. Como sabemos, no tiene ninguna posibilidad el modelo propugnado por el Estatuto catalán de 2006, que se demostró incompatible con los principios constitucionales, enunciados por el Tribunal Constitucional en la sentencia 31/2010.

A pesar de todo esto, hay que tener en cuenta la situación de Andalucía, que llevó a cabo una reforma de su Estatuto influenciada por el de Cataluña, antes de la sentencia del Tribunal Constitucional; de hecho el nuevo Estatuto andaluz, dispone formas de negociación bilateral en materia financiera entre el Estado y la CA, en una Comisión Mixta (art. 184). Esta regulación está actualmente vigente y puede producir todos sus efectos jurídicos, al no haber sido objeto de ninguna impugnación ante el TC mismo. Esta es la demostración de que el papel de la bilateralidad y su compatibilidad con la Constitución tiene que enfrentarse, además de la interpretación de los principios de la misma y de su integración con las normativas comunitarias, con las dinámicas de la política y de criterios de oportunidad también, como demostró la misma sentencia de Tribunal Supremo de 2004.

Por lo que concierne a su compatibilidad con el artículo 119 de la Constitución italiana (modificado por ley constitucional 1/2012), no puede descartarse una negociación bilateral: lo que será necesario será su previsión de forma compatible con el equilibrio de los presupuestos, de la solidaridad entre Regiones y de las obligaciones que derivan del ordenamiento europeo. Es decir un régimen de financiación armonizado y respetuoso con las obligaciones que esbozan un contexto que continúa siendo cada vez más multinivel.

TITLE: Fiscal autonomy: ¿cause or consequence of the regional speciality?

RESUMEN: Este trabajo realiza una comparación entre los sistemas de financiación de las autonomías o regiones en Italia y en España, haciendo especial hincapié en el caso del País Vasco y del Trentino / Alto Adige. El artículo se detiene en el análisis del principio de bilateralidad como eje articulador de las relaciones financieras en los dos Estados.

Palabras Clave: Federalismo Fiscal, bilateralidad, País Vasco, Trentino. ABSTRACT: This paper makes a comparison between the financial systems of the autonomous comunities of Spain and the regions of Italy with particular emphasis on the case of the Basque Country and the Trentino / 
Alto Adige. The article focuses on the analysis of the principle of bilateralism as the linchpin of financial relations in the two states.

KEY WORDS: Fiscal Federalism, bilateralism, Basque Country, Südtirol.

Recibido: 04.03.2013

Aceptado: 18.03.2013 